\title{
Exogenous stromal cell-derived factor-1 (SDF-1) suppresses the NLRP3 inflammasome and inhibits pyroptosis in synoviocytes from osteoarthritic joints via activation of the AMPK signaling pathway
}

\author{
Shuya Wang ${ }^{1}\left[\right.$ - Ali Mobasheri ${ }^{2,3,4,5}$ - Yue Zhang ${ }^{1} \cdot$ Yanli Wang ${ }^{1} \cdot$ Tianqi Dai $^{1} \cdot$ Zhiyi Zhang $^{1}$
}

Received: 18 February 2021 / Accepted: 24 April 2021 / Published online: 3 June 2021

(c) The Author(s) 2021

\begin{abstract}
Objective NLRP3 inflammasome may play a key role in OA pathogenesis. Stromal cell-derived factor-1 (SDF-1) is a homeostatic CXC chemokine. Since the role of SDF-1 in OA has not been explored, this study aimed to examine the effect of SDF-1 on NLRP3 inflammasome and pyroptosis in synoviocytes from OA joints.

Materials and methods Human synovium was obtained from OA patients for isolation of primary synoviocytes and a murine model of collagenase-induced OA was established for testing intra-articular injections of SDF-1. Immunoblotting assays were used to examine the effects and underlying mechanism of action of SDF-1 on NLRP3 inflammasome and synoviocyte pyroptosis in synoviocytes. Inhibitors of AMPK and PI3K-mTOR were utilized to investigate the key signaling pathways involved in SDF-1-mediated OA inflammasome formation and pyroptosis.

Results Synoviocytes from OA joints exhibited significantly higher expression of NLRP3 inflammasome and biomarkers of synoviocyte pyroptosis relative to healthy individuals. This was confirmed in the collagenase-induced OA model, where OA synoviocytes had a significantly lower SDF-1 expression than healthy ones. SDF-1 treatment in synoviocytes of OA patients and collagenase-induced OA led to significant downregulation in the expression of NLRP3 inflammasome and synoviocyte pyroptosis biomarkers. Inhibition of the AMPK signaling pathway significantly suppressed the inhibitory effect of SDF-1 on NLRP3 inflammasome expression of OA synoviocytes. However, blocking the SDF-1-activated PI3K-mTOR signaling pathway could still suppress the expression of NLRP3 inflammasome and synoviocyte pyroptosis biomarkers.

Conclusions SDF-1 ameliorates NLRP3 inflammasome and pyroptosis in OA synoviocytes through activation of the AMPK signaling pathway. Therefore, SDF-1 may be a novel therapeutic target for OA.
\end{abstract}

Keywords Osteoarthritis (OA) · Low-grade inflammation · Stromal cell-derived factor-1 - NLRP3 inflammasome · Pyroptosis $\cdot$ Synoviocyte $\cdot$ Synovitis

Ali Mobasheri

ali.mobasheri@oulu.fi

$\checkmark$ Zhiyi Zhang

zhangzhiyi2014@163.com

1 Department of Rheumatology, The First Affiliated Hospital of Harbin Medical University, No. 23 Youzheng St, Harbin 150001, China

2 Department of Joint Surgery, First Affiliated Hospital of Sun Yat-sen University, Guangzhou 510080, Guangdong, China
3 Research Unit of Medical Imaging, Physics and Technology, Faculty of Medicine, University of Oulu, PO Box 5000, 90014 Oulu, Finland

4 Department of Regenerative Medicine, State Research Institute Centre for Innovative Medicine, 08406 Vilnius, Lithuania

5 Department of Orthopedics, Rheumatology and Clinical Immunology, University Medical Center Utrecht, 508 GA Utrecht, The Netherlands 


\section{Introduction}

Osteoarthritis (OA) is the most common form of arthritis and a highly prevalent and disabling condition that affects movable joints, such as knees, hips, and hands (Katz et al. 2021). Currently, OA is considered to be a whole joint disease, as opposed to a simple cartilage wear and tear disease (Deveza and Loeser 2018). The pathogenesis of OA involves mechanical (Hunt et al. 2020), metabolic (Zheng et al. 2021), and inflammatory factors (Hunter and BiermaZeinstra 2019; Chow and Chin 2020). Recent research suggests that low-grade inflammation plays an important role in the development and progression of OA (Scanzello 2017). There are no effective disease-modifying osteoarthritis drugs (DMOADs) which have been approved by the regulatory agency (Oo et al. 2018); therefore, we urgently need to find new targets for the development of effective therapeutics.

Stromal cell-derived factor-1 (SDF-1), also known as CXCL12, is a member of the homeostatic CXC family of chemokines, with emerging roles in bones, joints, and muscles (Gilbert et al. 2019). However, the role of SDF-1 in the pathogenesis of OA has yet to be determined. Recent research has showed that SDF-1 levels increase significantly in OA synovial fluid (Kanbe et al. 2002). Previous studies have speculated that SDF-1 may play a protective role in $\mathrm{OA}$ by inducing significant extents of osteoblast proliferation and upregulating the expression of collagen type I in OA patients (Lisignoli et al. 2006; Shen et al. 2014). We have previously reported that SDF-1 can increase vascular endothelial growth factor expression in chondrogenic progenitor cells (Wang et al. 2017). However, thus far, no study has been conducted to examine the effect of SDF-1 on the NLRP3 inflammasome and pyroptosis in OA or explored its underlying mechanism of action.

The inflammasome can promote inflammation by activating the cysteinyl aspartate specific proteinase (caspase-1) protease, a pro-inflammatory caspase and a key regulator of cell death (Martinon and Tschopp 2007). The NLRP3 inflammasome is known to be activated in a twostep process (Tschopp and Schroder 2010; Jo et al. 2016). First, toll-like receptors recognize endogenous material or microbial materials and trigger increased expression of pro-interleukin-(IL) $1 \beta$, pro-IL-18, and NLRP3 (Sandanger et al. 2013; Kelley et al. 2019). In the second step, an activation signal initiates NLRP3 oligomerization, which further recruits NLRP3, adapter protein apoptosis-associated speck-like protein (ASC), and pro-caspase-1 to form the inflammasome (Kelley et al. 2019). These processes result in the conversion of pro-caspase-1 to caspase-1, which, in turn, proteolytically cleaves pro-IL-18 and pro-IL-1 $\beta$ and subsequently triggering their activation and release
(Franchi et al. 2009). Finally, this step induces pyroptosis through cleavage of gasdermin D (GSDMD) (Shi et al. 2017). A recent study reported the involvement of the NLRP3 inflammasome in the pathogenesis of OA (McAllister et al. 2018). In addition, IL-1 $\beta$ synthesized by synoviocytes from the inflamed synovium have been shown to exacerbate the pro-degradative mechanisms responsible for cartilage breakdown (Sellam and Berenbaum 2010). This highlights the importance of the synovium in driving inflammatory processes within the joint in OA (Scanzello and Goldring 2012; Liu-Bryan 2013).

OA is a complex and serious disease (Hawker 2019) and there are no effective drugs available for its treatment. The NLRP3 inflammasome plays a unique role in the pathogenesis of synovitis (Jin et al. 2011), which is an important mechanism underlying OA pathogenesis (Scanzello and Goldring 2012). To the best of our knowledge, no experimental study has yet examined the effects of SDF-1 on the NLRP3 inflammasome and pyroptosis in OA. We hypothesized that SDF-1 can decrease expression of the NLRP3 inflammasome and related proteins in synoviocytes from OA joints exhibiting synovitis and may have potential as a novel therapeutic target for synovitis in OA. Therefore, we utilized recombinant SDF-1 to treat fibroblast-like synoviocytes (FLS) from human OA joints and synoviocytes derived from collagenase-induced OA mice and explored the effects and underlying mechanism of action of SDF-1 in these in vitro and preclinical models of inflammatory OA.

\section{Materials and methods}

\section{Materials}

SDF-1 (purity is $>97 \%$, by SDS-PAGE under reducing conditions and visualized by silver stain) was purchased from R\&D Systems. Dorsomorphin (Compound C, CC), inhibitor of phosphatidylinositol 3-kinase (PI3K)-mammalian target of rapamycin (mTOR), and 3-methyladenine (3MA) were purchased from MedChemExpress. Anti-NLRP3, anti-caspase-1, anti-IL-1 $\beta$, and anti-PI3K antibodies were purchased from Abcam. Anti-ASC antibody was purchased from Santa Cruz Biotechnology. Anti-GSDMD and anti-microtubuleassociated protein light chain 3 (LC3) antibodies were purchased from Cell Signaling Technology. Anti-phospho-adenosine 5'-monophosphate-activated protein kinase (AMPK) $\alpha 1 / 2$, anti-GAPDH, horseradish peroxidase (HRP) goat anti-rabbit IgG, and HRP goat anti-mouse IgG antibodies were purchased from Abbkine. Anti-mTOR antibody was purchased from Bioworld Technology. Anti-p62 antibody was purchased from ABclonal. 


\section{In vitro culture of healthy and OA FLS and SDF-1 stimulation of OA FLS}

Healthy primary FLS were purchased from Procell. Synovium was isolated from the knee of OA patients at the time of all knee replacement surgery carried out in the Department of Orthopaedics, the First Affiliated Hospital of Harbin Medical University. Knee OA was diagnosed according to the criteria established by the American College of Rheumatology (Altman et al. 1986). Informed consent was obtained from all patients participating in the study. Synovial tissues were washed three times with phosphate-buffered saline (PBS), cut into sections with an area of $2-3 \mathrm{~mm}^{2}$, and digested in $2 \mathrm{mg} / \mathrm{ml}$ collagenase type II (Sigma-Aldrich) at $37{ }^{\circ} \mathrm{C}$ overnight and isolated cells were filtered through a cell strainer (Alvaro-Gracia et al. 1990). Healthy human FLS and OA-derived FLS were used between 4 and 6 passages. OA FLS were treated with SDF-1 $(20,50$, or $100 \mathrm{ng} / \mathrm{ml})$ for $24 \mathrm{~h}$, or SDF-1 (100 ng/ml) for 1, 3, 6, 12, or $24 \mathrm{~h}$. To identify the signaling pathways involved in the effect of SDF-1, OA-derived FLS were pretreated with the AMPK inhibitor CC $(10 \mu \mathrm{mol} / \mathrm{ml})$ for $1 \mathrm{~h}$ or the PI3K-mTOR inhibitor $3 \mathrm{MA}(5 \mathrm{mM})$ for $2 \mathrm{~h}$ prior to the administration of SDF-1 $(100 \mathrm{ng} / \mathrm{ml})$.

\section{In vitro proliferation assay}

Proliferation of OA-derived FLS was evaluated using the Cell Counting Kit-8 (CCK-8, Dojindo Molecular Technologies), as described previously (Wang et al. 2019). Briefly, $10^{4}$ cells were seeded in a 96-well plate. After $48 \mathrm{~h}$, these cells were treated with different concentrations of SDF-1 added to fresh medium and incubated at $37^{\circ} \mathrm{C}$ for $24 \mathrm{~h}$. The plates were then read at a wavelength of $450 \mathrm{~nm}$. The experiment was repeated three times.

\section{Gene expression analysis}

Total RNA was extracted from OA-derived FLS with the TRIzol reagent (Invitrogen) and reverse-transcribed to cDNA. Real-time PCR analysis was carried out using TOYOBO reverse transcription reagents and SYBR Green PCR Master Mix (Bio-Rad). Specific primers were custom synthesized from Ruibiotech (Supplementary Table 1). GAPDH was used as a reference gene. All samples were measured in triplicates and the relative quantification method $\left(2^{-\Delta \Delta C_{\mathrm{T}}}\right)$ was used to calculate the relative expression levels of genes in the different groups (Wang et al. 2017).

\section{Western blotting}

Protein expression levels in OA-derived FLS were estimated by western blotting, as previously described with some modifications (Zhang et al. 2015). Total protein from healthy or OA FLS cultures was extracted with cold RIPA lysis buffer and Bio-Rad assayed. Extracted proteins were then separated using 12.5 or $7.5 \%$ sodium dodecyl sulfate-polyacrylamide gel electrophoresis (SDS-PAGE) and transferred to polyvinylidene fluoride (PVDF) membranes. The blots were probed with anti-NLRP3 (1:1000), anti-ASC (1:800), anti-caspase-1 (1:1000), anti-GSDMD (1:1000), anti-IL-1 $\beta$ (1:1000), anti-phosphorylated-AMPK (1:1000), anti-phosphorylated-PI3K (1:1000), anti-mTOR (1:1000), anti-LC3 (1:1000), anti-p62 (1:1000), and anti-GAPDH (1:5000) antibodies at $4{ }^{\circ} \mathrm{C}$ overnight, followed by incubation with an HRP-conjugated goat anti-rabbit or goat anti-mouse IgG secondary antibody $(1: 10,000)$ for $2 \mathrm{~h}$ at room temperature. The labeled blots were finally imaged and analyzed using the ImageJ software (National Institutes of Health, Bethesda, USA).

\section{Establishment of collagenase-induced OA model and SDF-1 treatment}

Twelve-week-old male C57BL/6 mice were obtained from the Second Affiliated Hospital of Harbin Medical University Animal Centre (Harbin, China) and maintained at the Laboratory Animal Centre, the First Affiliated Hospital of Harbin Medical University. Mice were housed with free access to water and food. Animal experiments were performed according to the guidelines of the National Institutes of Health for the Care and Use of Laboratory Animals (Zhang et al. 2015). The study was conducted in accordance with the Declaration of Helsinki and was approved by the Ethics Committee of the First Affiliated Hospital of Harbin Medical University (IRB: 2018139). A collagenase-induced OA mouse model was established as previously described, with minor modifications (van der Kraan et al. 1990). Briefly, OA was induced in mice using two intra-articular injections of $5 \mathrm{U}$ collagenase type VII (Sigma-Aldrich) on day 0 and day 2 in the right knee. Administration of collagenase type VII is known to damage the cruciate and collateral ligaments, leading to instability of knee joints, and finally resulting in chronic synovial activation and cartilage destruction, which represented an OA-like phenotype. Then, SDF-1 (120 ng/kg) was injected twice a week in the knee joint of collagenase-induced OA mice from day 7. Day 42 represented the end point of the establishment of the disease model. The control group consisted of knee joints injected with saline ( $n=3$ for each group).

\section{Microcomputed tomography (micro-CT) imaging}

On day 42 following the initial collagenase VII injection, mice were killed. The distal femur and proximal tibia were cut with blunt scissors to obtain knee joint tissues and scanned with a Quantum GX microcomputed tomography 
(micro-CT) imaging system (Perkin Elmer, Waltham, MA, USA) (Wang et al. 2019) to investigate the effect of SDF-1 on the three-dimensional (3D) bone structure in collagenase-induced OA mice. The voxel size was $20 \mu \mathrm{m}$, $\mathrm{X}$-ray tube voltage was $70 \mathrm{kV}$, current was set to $88 \mu \mathrm{A}$, and the exposure time was $14 \mathrm{~min}$. The acquired images were reconstructed into a 3D representation to exhibit structural changes induced by OA.

\section{Haematoxylin and eosin (H\&E) staining}

Whole knee joints were collected and fixed in $4 \%$ formalin, decalcified in $4 \%$ formic acid in PBS, and then decalcified in $10 \%$ ethylenediaminetetraacetic acid (Sigma-Aldrich, Shanghai, China) for 4 weeks. The tissue was dehydrated in a solvent system containing increasing amount of alcohol, and then embedded in paraffin. Coronal sections (5- $\mu \mathrm{m}$ thickness) were cut and stained with hematoxylin and eosin (H\&E). Two independent observers scored the histological changes based on synovial hyperplasia, joint inflammation, and bone erosion (Wang et al. 2019).

\section{Immunohistochemistry (IHC)}

Immunostaining of knee joint sections was performed with specific antibodies against target proteins according to a previously published protocol with some modifications (Wang et al. 2019). Sections were incubated with antiNLRP3 (1:100), anti-ASC (1:100), anti-caspase-1 (Wanleibio, 1:100), anti-GSDMD (Affinity Biosciences, 1:100), and anti-IL-1 $\beta(1: 100)$ antibodies overnight at $4{ }^{\circ} \mathrm{C}$. On the following day, these tissue sections were incubated with a polymer-HRP detection system (PV9001, ZSGB-BIO) and visualized with a diaminobenzidine (DAB) peroxidase substrate kit (ZLI-9017, ZSGB-BIO). Each section was evaluated under a microscope (DMi8, LEICA) at $20 \times$ magnification. Histological analyses were performed in a blinded manner by two independent observers. Image-ProPlus6 software 6.0 (Media Cybernetics, Inc.) was used to analyze the average integrated optical density (IOD) of three randomly selected areas of the acquired images.

\section{Statistical analysis}

All data collected are represented as mean \pm standard error of the mean (SEM). GraphPad Prism 5.0 software (San Diego, CA, USA) was used for statistical analysis. Data were checked for statistical significance using Student's $t$-test or one-way analysis of variance (ANOVA). Values of $p<0.05$ were considered to indicate a statistically significant difference.

\section{Results}

\section{SDF-1 suppressed NLRP3 inflammasome and pyroptosis of OA FLS}

OA-derived FLS exhibited a significantly higher expression of the NLRP3 inflammasome and pyroptosis-related proteins, GSDMD and IL- $1 \beta$, compared to healthy synoviocytes $(p<0.05)$. The expression levels of NLRP3 inflammasome (NLRP3, ASC, and caspase-1), GSDMD, and IL-1 $\beta$ were significantly upregulated in OA FLS compared with that in healthy FLS $(p<0.05, n=9)$, as estimated by western blotting (Fig. 1a). OA FLS also had a significantly lower gene expression of SDF-1 $(p<0.05$, $n=5$ ) compared to healthy FLS (Fig. 1b).

To further study the effect of SDF-1 on OA FLS inflammasome and pyroptosis, a series of experiments were carried out. First, to rule out any cytotoxic effects, we examined the influence, if any, of SDF-1 on the proliferation of OA FLS. Only SDF-1 at a concentration of $20 \mathrm{ng} / \mathrm{ml}$ was found to increase the proliferation of OA FLS $(p<0.05, n=6)$; the other two concentrations of SDF-1 used in these experiments had no significant influence on cell proliferation $(p>0.05)$ (Fig. 1c). OA FLS were examined after treatment with different doses of SDF-1 (20, 50, and $100 \mathrm{ng} / \mathrm{ml})$. Gene (Fig. 1d) and protein (Fig. 1e) expression levels of the NLRP3 inflammasome (NLRP3, ASC, and caspase-1), GSDMD, and IL-1 $\beta$ were significantly downregulated on SDF-1 treatment, with the maximal effect observed at a $100 \mathrm{ng} / \mathrm{ml}$ concentration $(p<0.05, n=6-8)$.

\section{SDF-1 decreased NLRP3 inflammasome and pyroptosis in collagenase-induced $O A$ mouse model}

To examine the effect of SDF-1 in vivo, we treated collagenase-induced OA mice by intra-articular injection of SDF-1 (recombinant human SDF-1 was reconstituted in sterile PBS containing at least $0.1 \%$ bovine serum albumin, and the treatment dosage of SDF-1 used was $240 \mathrm{ng} /$ $\mathrm{kg}$ per week). Micro-CT results showed that at week 7 of the treatment, collagenase-induced OA mice exhibited narrowing joint space and osteophyte formation. Alongside type VII collagenase injection for a week, further SDF-1 treatment was observed to delay these pathological phenotypes. These results could also be confirmed from inspection of the H\&E stained tissue sections. Furthermore, IHC staining also showed that collagenase-induced OA mice had a significantly higher expression of NLRP3, ASC, caspase-1, GSDMD, and IL-1 $\beta$ than mice in the control group 
A

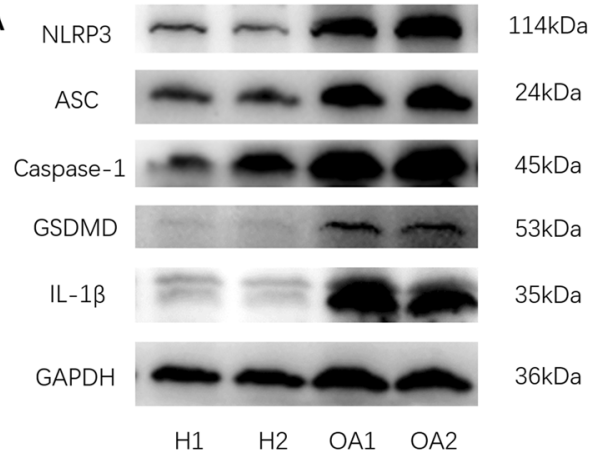

B
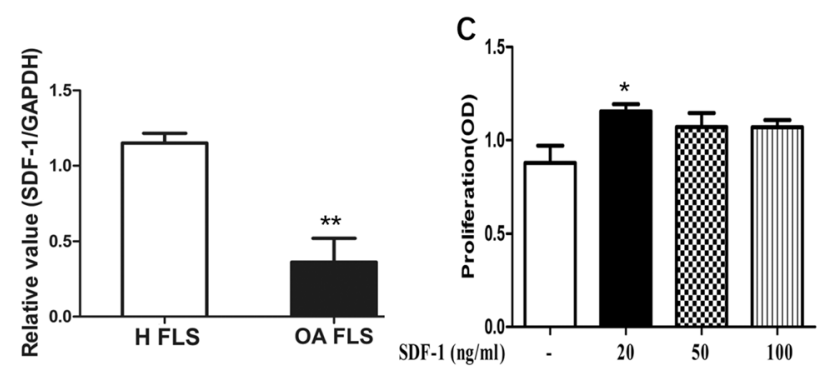

E

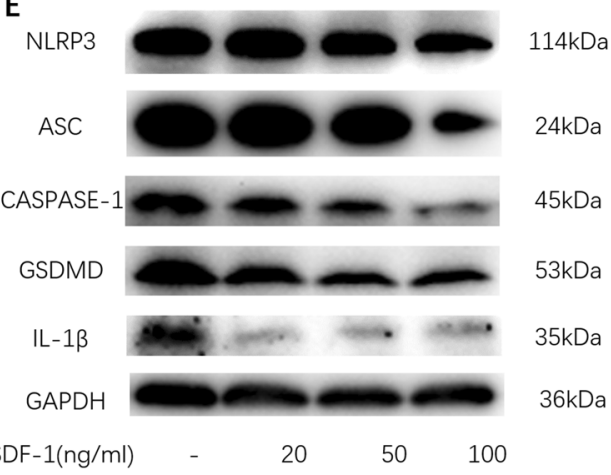

Fig. 1 SDF-1 suppressed NLRP3 inflammasome and pyroptosis of OA FLS. NLRP3, ASC, caspase-1, GSDMD and IL-1 $\beta$ protein expression in healthy FLS and OA FLS were estimated by western blotting $(\mathbf{a}, n=9)$. Data are expressed as means \pm standard error of the mean (SEM). In addition, SDF-1 gene expressions in healthy FLS and OA FLS were detected by RT-PCR $(\mathbf{b}, n=5)$. After treatment with different concentrations of SDF-1 $(20,50$, and $100 \mathrm{ng} / \mathrm{ml})$

$(p<0.05)$. Conversely, SDF-1 treatment decreased their protein level expression $(p<0.05)$ (Fig. 2).

\section{SDF-1 suppressed NLRP3 inflammasome expression and pyroptosis of OA FLS through AMPK signaling pathway}

Next, we explored how SDF-1 contributed to the inhibitory effect mentioned earlier. First, our results showed that SDF-1 $(100 \mathrm{ng} / \mathrm{ml})$ increased the expression of phosphorylated-AMPK (p-AMPK), indicating that SDF-1 activates the AMPK signaling pathway. This effect reached a peak
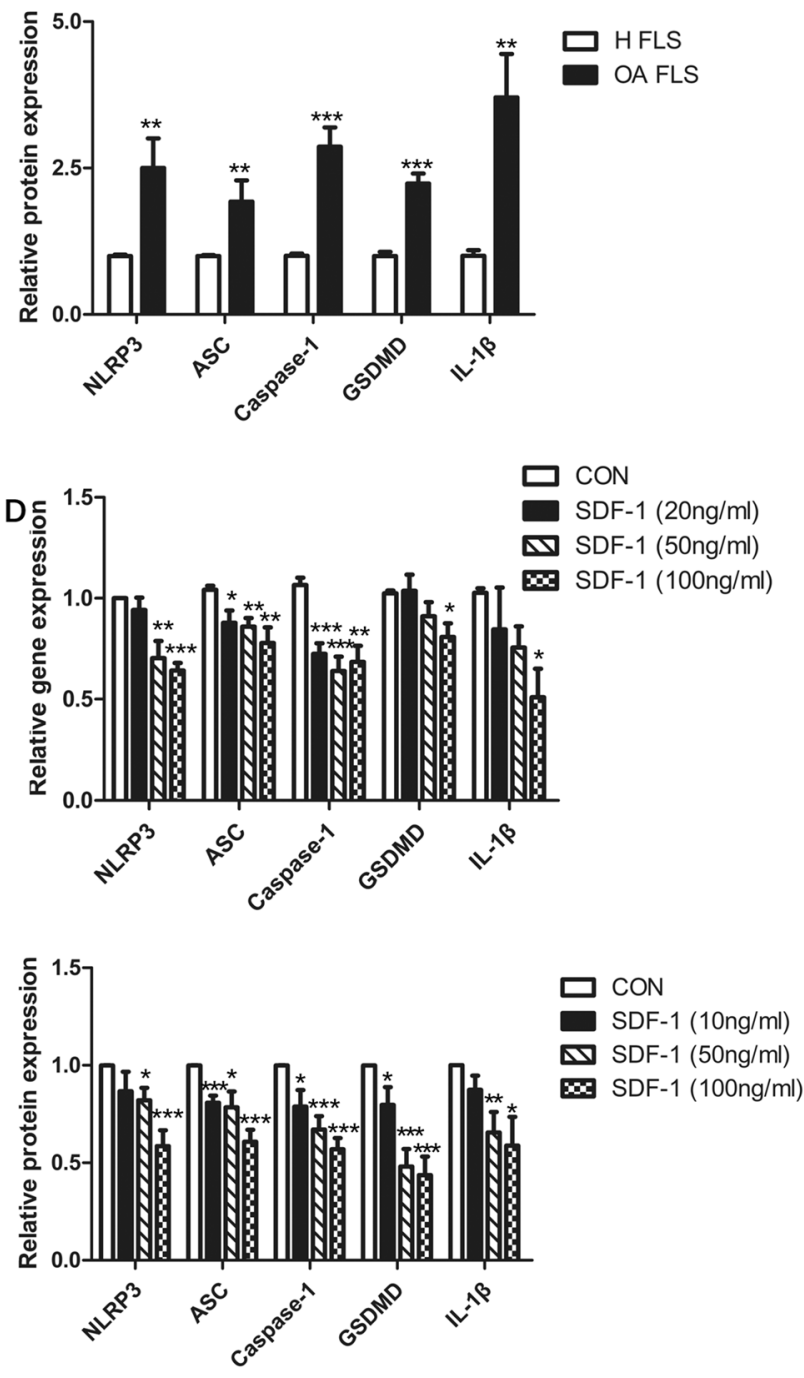

for $24 \mathrm{~h}(n=6)$, CCK-8 was added to OA FLS and incubated for $1 \mathrm{~h}$. Absorbance (OD, optical density value) at $450 \mathrm{~nm}$ was detected by a BioTek microplate reader (c) (OD, optical density value). SDF-1 downregulated the gene (d) and protein (e) expression levels of NLRP3 inflammasome, GSDMD, and IL-1 $\beta$, which represented pyroptosis $(p<0.05, n=6-8)$. * $p<0.05 ; * * p<0.01 ; * * * p<0.001$ versus control; CON, control: OA FLS treated only with the medium

at the $3 \mathrm{~h}$ time point after injection. After treatment with SDF-1 $(100 \mathrm{ng} / \mathrm{ml})$ for more than $6 \mathrm{~h}$, the activity of the AMPK pathway decreased (Fig. $3 a)(p<0.05, n=8)$. OA FLS were then treated with SDF-1 $(100 \mathrm{ng} / \mathrm{ml})$ for $24 \mathrm{~h}$ with/out pre-treatment with the inhibitor of AMPK signaling pathway $\mathrm{CC}(10 \mu \mathrm{M})$ for $1 \mathrm{~h}$. CC treatment could reverse the inhibitory effect of SDF-1 on the expression of NLRP3 inflammasome in OA FLS (Fig. 3b) $(p<0.05$, $n=6$ ). This revealed that the AMPK signaling pathway plays an important role in the function of SDF-1. 


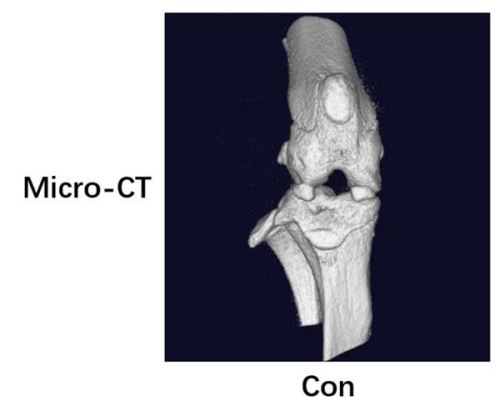

Con

HE

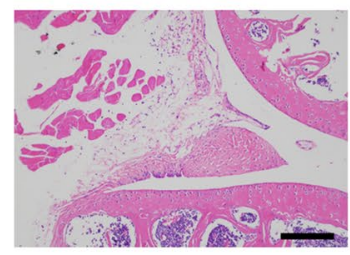

Con

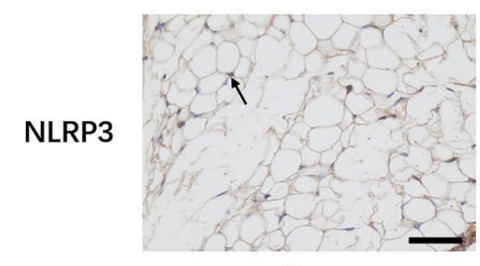

Con

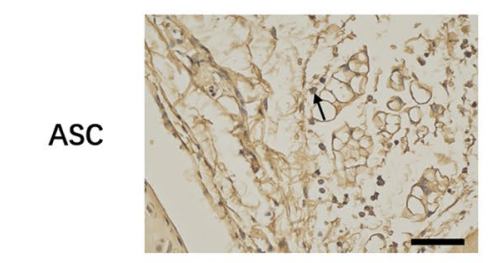

Con

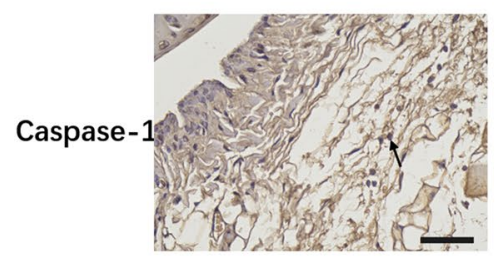

Con

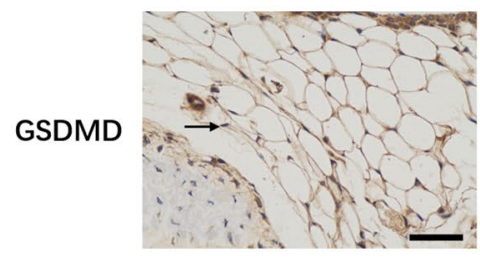

Con

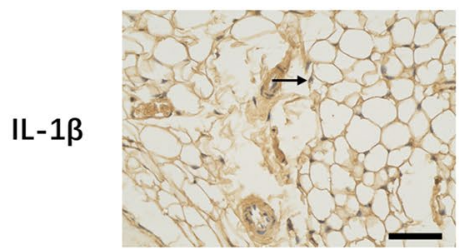

Con

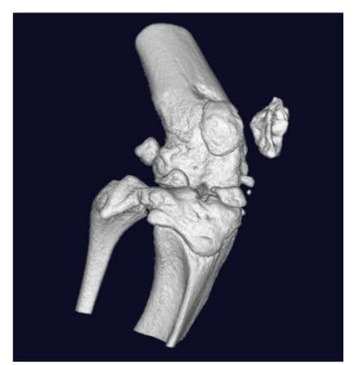

CIOA

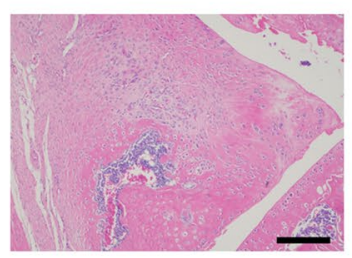

CIOA

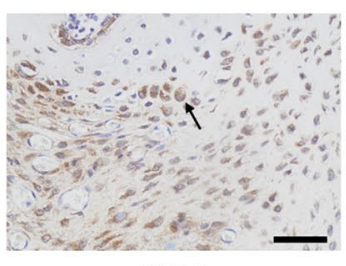

CIOA

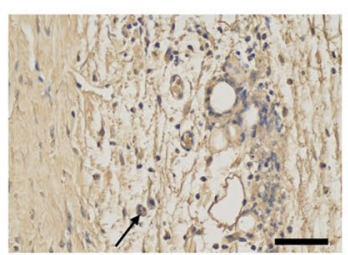

CIOA

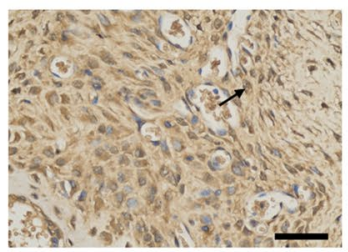

CIOA

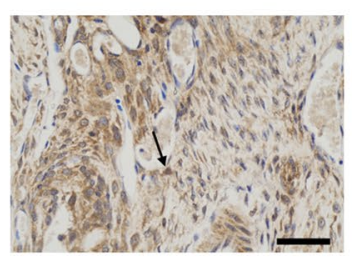

CIOA

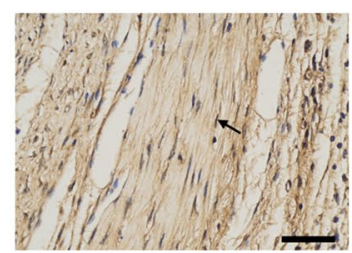

CIOA

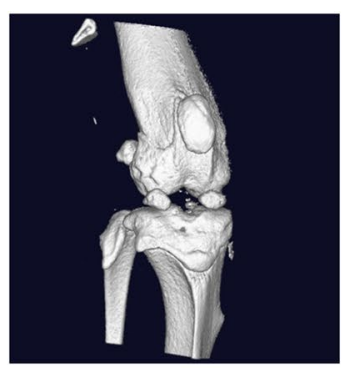

$\mathrm{CIOA}+\mathrm{SDF}-1$

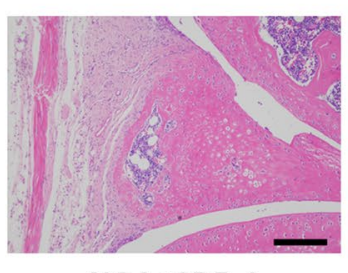

$\mathrm{ClOA}+\mathrm{SDF}-1$

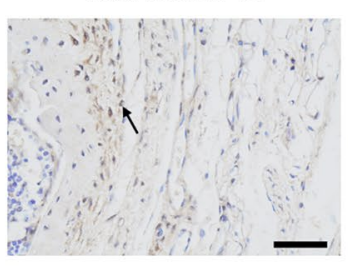

$\mathrm{CIOA}+\mathrm{SDF}-1$

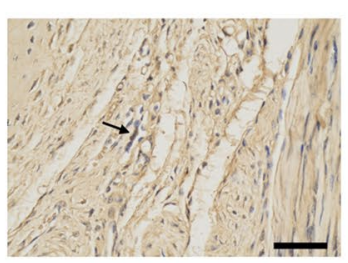

CIOA+SDF-1

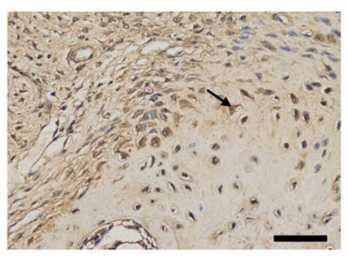

$\mathrm{CIOA}+\mathrm{SDF}-1$

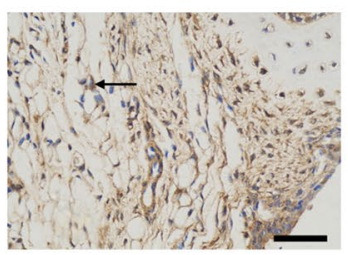

CIOA+SDF-1

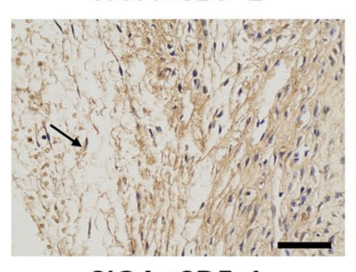

$\mathrm{ClOA}+\mathrm{SDF}-1$
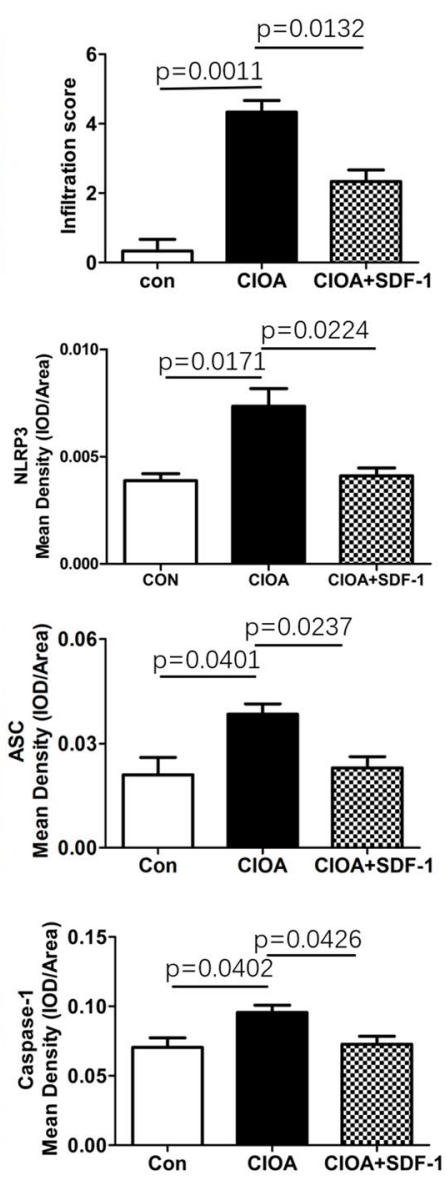

$\mathrm{p}=0.0096$
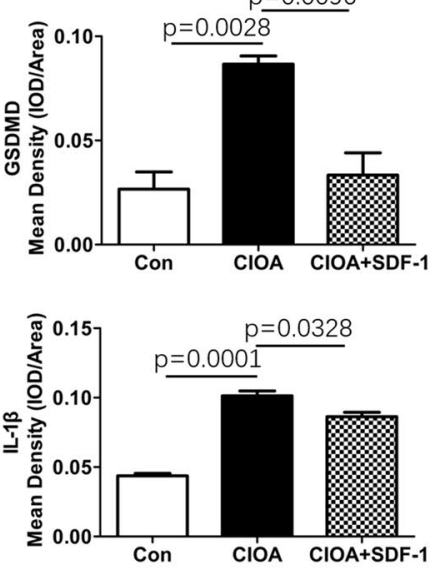
4Fig. 2 SDF-1 decreased NLRP3 inflammasome in collagenaseinduced OA mouse model. Type VII collagenase injection can cause narrowing of the joint space and osteophyte formation in Micro-CT and $\mathrm{H} \& \mathrm{E}$ staining. However, intra-articular injection of $120 \mathrm{ng} / \mathrm{kg}$ SDF-1 twice a week delayed the pathological progression of these symptoms. IHC results showed that the synovium of collagenaseinduced OA mice had a significantly higher expression of NLRP3, ASC, caspase-1, and protein markers of pyroptosis (GSDMD and IL- $1 \beta)$ compared to the synovium of control mice $(p<0.05)(n=3)$; however, SDF-1 treatment could reverse this effect to some extent. IOD, integrated optical density

\section{Role of PI3K-mTOR signaling pathway in SDF-1-mediated suppression of NLRP3 inflammasome expression in OA FLS}

Furthermore, to explore the contribution of the AMPK signaling pathway, we studied the influence of SDF-1 in the PI3K-mTOR signaling pathway. Our results showed that SDF-1 increased the protein levels of p-PI3K and LC3II/I (Fig. 4a, b), and decreased protein levels of mTOR and p62 (Fig. 4b). The effect of SDF-1 on p-PI3K was also found to peak at $3 \mathrm{~h}$. This means that SDF-1 activates both the PI3K-mTOR signaling and autophagic pathways. We also treated OA FLS with 3MA before SDF-1 treatment.
Unexpectedly, inhibition of the PI3K-mTOR signaling pathway by 3MA (3-methyladenine, 3-MA, is a highly selective inhibitor of autophagy through PI3K signaling pathway) could not sufficiently reverse the inhibitory effect of SDF-1 on NLRP3 inflammasome and pyroptosis of OA synoviocytes (Fig. 4c).

\section{Discussion}

OA is a complex chronic disease and synovitis plays a prominent role in OA pathogenesis (Han et al. 2020). The NLRP3 inflammasome is also known to be associated with synovitis (Clavijo-Cornejo et al. 2016). However, no previously published study has explored whether SDF-1 may exert an effect on NLRP3 inflammasome and pyroptosis in OA or investigated the underlying mechanism of action, if any. Our results suggest that OA synoviocytes had significantly higher expression of NLRP3 inflammasome biomarkers (NLRP3, ASC, and caspase-1) and pyroptosis-related proteins (GSDMD and IL-1 $\beta$ ) compared to healthy synoviocytes. These results were consistent with those of a previous study (Clavijo-Cornejo et al. 2016). Meanwhile, we also observed that OA FLS was characterized by a significantly lower gene

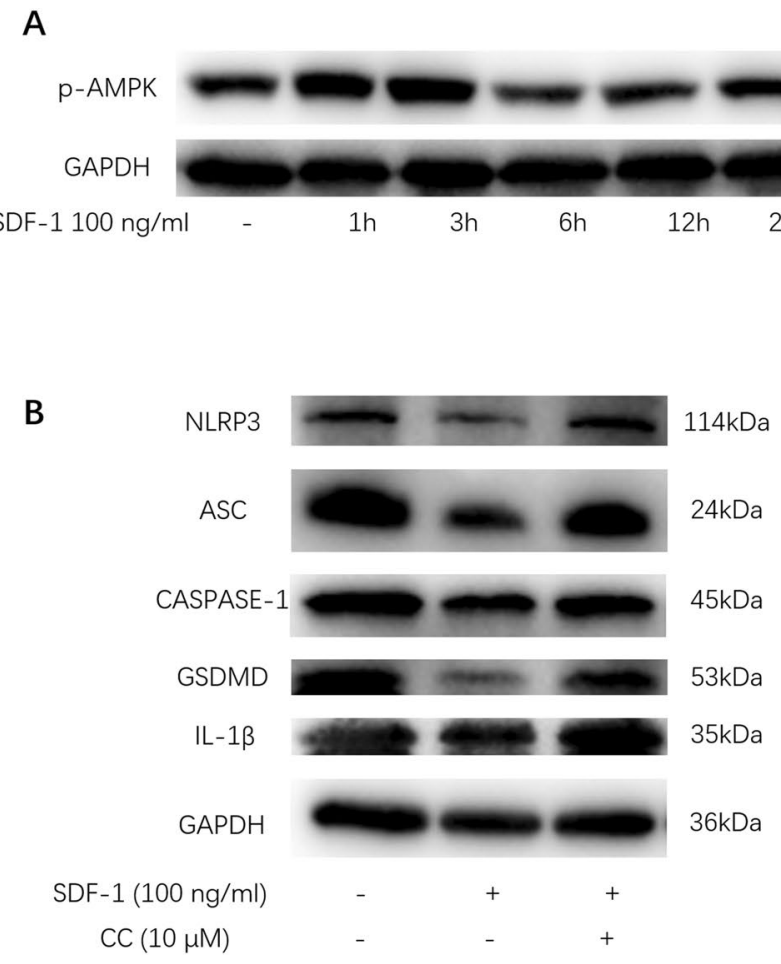

Fig. 3 SDF-1 suppressed expression of NLRP3 inflammasome and pyroptosis in OA FLS through AMPK signaling pathway. SDF-1 was found to activate the AMPK signaling pathway at 1-, 3-, and 6-h time points, with the maximal change observed at $3 \mathrm{~h}(\mathbf{a})(n=8)$. Pretreatment of OA FLS with $10 \mu \mathrm{M}$ CC (an AMPK signaling pathway
$60 \mathrm{kDa}$

$36 \mathrm{kDa}$
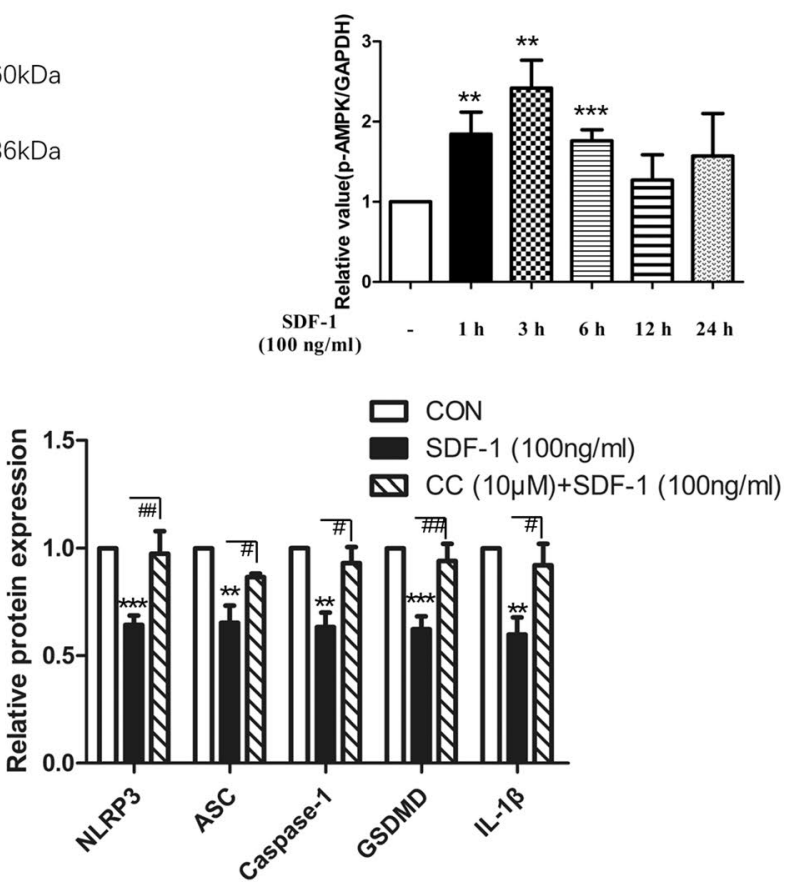

inhibitor) for $1 \mathrm{~h}$ led to suppression of the inhibitory effect of SDF-1 on NLRP3 inflammasome and pyroptosis (b) $(n=6) .{ }^{*} p<0.05$; $*^{*} p<0.01 ; * * * p<0.001$ versus control; CON, control: OA FLS treated with only the medium; \#p $<0.05$; \#\#p $<0.01$; \#\# $p<0.001$ versus SDF-1 $(100 \mathrm{ng} / \mathrm{ml})$ treatment 

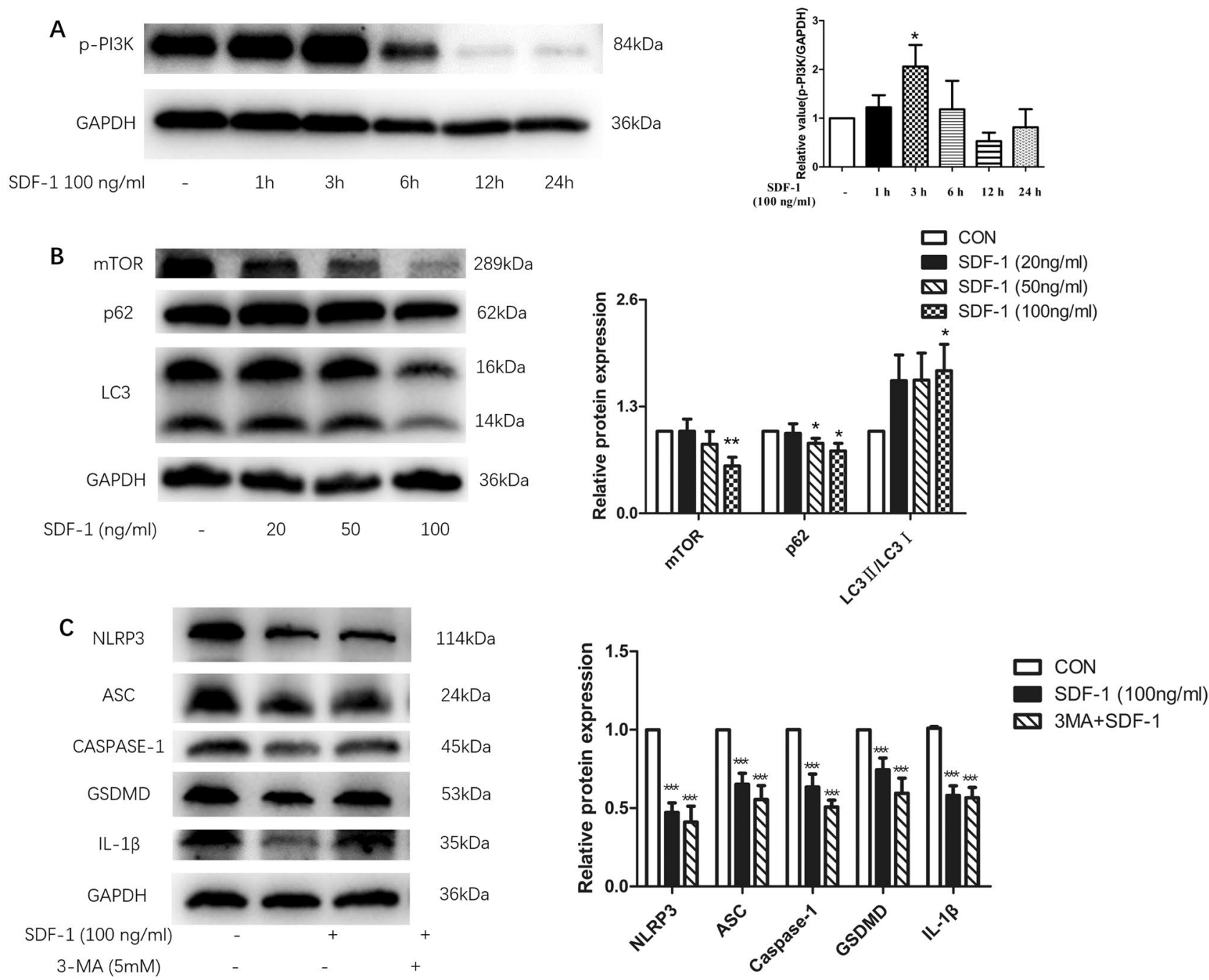

Fig. 4 Downregulation of OA FLS inflammasome and pyroptosis by SDF-1 is not mediated by the PI3K-mTOR signaling pathway. SDF-1 activated the PI3K signaling pathway at $3 \mathrm{~h}(\mathbf{a})(n=6)$. Besides, SDF-1 $(100 \mathrm{ng} / \mathrm{ml})$ decreased the protein expression of mTOR and

p62 in OA FLS and increased the protein expression level of LC3II/I (b) $(n=6-12)$. Pre-treated OA FLS with $5 \mathrm{mM} \mathrm{3MA} \mathrm{(inhibitor}$ of PI3K-mTOR) for $2 \mathrm{~h}$ could not reverse the inhibitory effects of SDF-1 (c) $(n=6) .{ }^{*} p<0.05 ; * * p<0.01 ; * * * p<0.001$ versus controls

expression of SDF-1 compared to that in healthy FLS. We then explored if SDF-1 treatment could slow down OA pathogenesis, and indeed observed decreased expression of NLRP3 inflammasome biomarkers in the presence of SDF-1. Collectively, our observations indicate that SDF-1 may have exerted some beneficial effect on the collagenase-induced OA model. These functions of SDF-1 have been confirmed, in part, by another indirect study. Intrathecal application of SDF-1a could regulate NLRP3 inflammasome expression in spinal cord injury model (Zendedel et al. 2016).

To the best of our knowledge, this is the first report on the effect and underlying mechanism of action of SDF-1 on the expression of NLRP3 inflammasome in OA synoviocytes. Results from phosphospecific immunoblotting assay confirmed that SDF-1 could activate the AMPK and PI3K-mTOR

signaling pathways. In addition, SDF-1-mediated downregulation of NLRP3 inflammasome, GSDMD, and IL- $1 \beta$ was substantially blocked only by the AMPK inhibitor (CC), but not by the PI3K-mTOR signaling pathway inhibitor (3MA). These results revealed that SDF-1 inhibits the OA FLS NLRP3 inflammasome and pyroptosis through the AMPK signaling pathway, while the PI3K-mTOR pathway counteracts the effect of SDF-1 (Supplementary Fig. 1). Based on these observations, we proposed a novel mechanism for SDF-1 in OA treatment. To the best of our knowledge, inhibitors of SDF-1 (such as AMD3100) have been previously reported to ameliorate degradation of OA cartilage (Lu et al. 2019). However, our study represents the first report confirming that treatment of human OA-derived primary synoviocytes with SDF-1 has these beneficial effects. This is relevant since differences in OA animal models or species-specific 
differences could account for variations in treatment efficacy in preclinical animal models and human patients. AMD3100 is known to be an inhibitor of the SDF-1 receptor CXC chemokine receptor 4 (CXCR4), while SDF-1 also binds to other receptors, such as CXCR7 and atypical chemokine receptor 3 (ACKR3) (Wang et al. 2018). Therefore, the effect of AMD3100 may not comprehensively represent the function of SDF-1 protein, especially in a clinical setting.

The limitations of the present study are as follows: first, we obtained synovial tissues from a limited number of patients with local OA; therefore, future studies will expand the sample size to generalize our findings to a much wider patient population. Second, these observations must be verified using alternative techniques, for example immunofluorescence and electron microscopy.

\section{Conclusion}

In summary, our results demonstrate that key biomarkers and molecular components of the NLRP3 inflammasome and pyroptosis exhibit significantly higher expression in OA FLS compared to healthy FLS. Consistent with these observations, the synovium of collagenase-induced OA mice was also shown to express significantly higher levels of these proteins compared to healthy synovium from control mice. The NLRP3 inflammasome and pyroptosis may, therefore, influence the course of disease progression and adversely affect OA patients. SDF-1 treatment could inhibit the NLRP3 inflammasome and pyroptosis of OA FLS and synovium of collagenase-induced OA mice. Furthermore, our investigation of downstream signaling pathways revealed that SDF-1 is a putative cytoprotective protein that suppresses OA FLS NLRP3 inflammasome and pyroptosis through the AMPK signaling pathway, with the PI3K-mTOR signaling pathway counteracting the effect of SDF-1. These novel observations represent, to the best of our knowledge, the first report of insights into the effects and underlying mechanism of action of SDF-1-mediated inhibition of the OA NLRP3 inflammasome and pyroptosis. The data presented establish a new line of evidence to support the potential suitability of SDF-1 as a novel experimental therapeutic and cytoprotective agent for treating OA.

Supplementary Information The online version contains supplementary material available at https://doi.org/10.1007/s10787-021-00814-x.

Acknowledgements This work was supported by grants from the National Natural Science Foundation of China to Zhiyi Zhang (NSFC 81771749) and Yue Zhang (NSFC81771748), and partially by the First Affiliated Hospital of HMU Merit Review Frontiers grant to Shuya Wang (HYD2020YQ0008) and Health and Health Commission of Heilongjiang Province grant to Yanli Wang (2019-013). Ali Mobasheri is supported by financial support from the European Structural and Social Funds (ES Struktūrinès Paramos) through the Research
Council of Lithuania (Lietuvos Mokslo Taryba) according to the activity 'Improvement of researchers' qualification by implementing world-class R\&D projects' of Measure No. 09.3.3-LMT-K-712 (grant application code: 09.3.3-LMT-K-712-01-0157, agreement No. DOTSUT-215) and the new funding program: Attracting Foreign Researchers for Research Implementation (2018-2022). We sincerely thank Dr. Juan Zhang, Ms. Lili Luo, Ms. Xueying Lang, and Ms. Xiaoying Zhu for their suggestions on this study and during the drafting of this article.

Funding Open access funding provided by University of Oulu including Oulu University Hospital.

Open Access This article is licensed under a Creative Commons Attribution 4.0 International License, which permits use, sharing, adaptation, distribution and reproduction in any medium or format, as long as you give appropriate credit to the original author(s) and the source, provide a link to the Creative Commons licence, and indicate if changes were made. The images or other third party material in this article are included in the article's Creative Commons licence, unless indicated otherwise in a credit line to the material. If material is not included in the article's Creative Commons licence and your intended use is not permitted by statutory regulation or exceeds the permitted use, you will need to obtain permission directly from the copyright holder. To view a copy of this licence, visit http://creativecommons.org/licenses/by/4.0/.

\section{References}

Altman R, Asch E, Bloch D, Bole G, Borenstein D, Brandt K, Christy W, Cooke TD, Greenwald R, Hochberg M et al (1986) Development of criteria for the classification and reporting of osteoarthritis. Classification of osteoarthritis of the knee. Diagnostic and Therapeutic Criteria Committee of the American Rheumatism Association. Arthritis Rheum 29:1039-1049

Alvaro-Gracia JM, Zvaifler NJ, Firestein GS (1990) Cytokines in chronic inflammatory arthritis. V. Mutual antagonism between interferon-gamma and tumor necrosis factor-alpha on HLA-DR expression, proliferation, collagenase production, and granulocyte macrophage colony-stimulating factor production by rheumatoid arthritis synoviocytes. J Clin Invest 86:1790-1798

Chow YY, Chin KY (2020) The role of inflammation in the pathogenesis of osteoarthritis. Mediators Inflamm 2020:8293921

Clavijo-Cornejo D, Martinez-Flores K, Silva-Luna K, Martinez-Nava GA, Fernandez-Torres J, Zamudio-Cuevas Y, Guadalupe Santamaria-Olmedo M, Granados-Montiel J, Pineda C, Lopez-Reyes A (2016) The overexpression of NALP3 inflammasome in knee osteoarthritis is associated with synovial membrane prolidase and NADPH oxidase 2. Oxid Med Cell Longev 2016:1472567

Deveza LA, Loeser RF (2018) Is osteoarthritis one disease or a collection of many? Rheumatology (Oxford) 57:iv34-iv42

Franchi L, Eigenbrod T, Munoz-Planillo R, Nunez G (2009) The inflammasome: a caspase-1-activation platform that regulates immune responses and disease pathogenesis. Nat Immunol $10: 241-247$

Gilbert W, Bragg R, Elmansi AM, McGee-Lawrence ME, Isales CM, Hamrick MW, Hill WD, Fulzele S (2019) Stromal cell-derived factor-1 (CXCL12) and its role in bone and muscle biology. Cytokine 123:154783

Han D, Fang Y, Tan X, Jiang H, Gong X, Wang X, Hong W, Tu J, Wei W (2020) The emerging role of fibroblast-like synoviocytes-mediated synovitis in osteoarthritis: an update. J Cell Mol Med 24:9518-9532 
Hawker GA (2019) Osteoarthritis is a serious disease. Clin Exp Rheumatol 37(Suppl 120):3-6

Hunt MA, Charlton JM, Esculier JF (2020) Osteoarthritis year in review 2019: mechanics. Osteoarthritis Cartilage 28:267-274

Hunter DJ, Bierma-Zeinstra S (2019) Osteoarthritis. Lancet 393:1745-1759

Jin C, Frayssinet P, Pelker R, Cwirka D, Hu B, Vignery A, Eisenbarth SC, Flavell RA (2011) NLRP3 inflammasome plays a critical role in the pathogenesis of hydroxyapatite-associated arthropathy. Proc Natl Acad Sci USA 108:14867-14872

Jo EK, Kim JK, Shin DM, Sasakawa C (2016) Molecular mechanisms regulating NLRP3 inflammasome activation. Cell Mol Immunol 13:148-159

Kanbe K, Takagishi K, Chen Q (2002) Stimulation of matrix metalloprotease 3 release from human chondrocytes by the interaction of stromal cell-derived factor 1 and CXC chemokine receptor 4 . Arthritis Rheum 46:130-137

Katz JN, Arant KR, Loeser RF (2021) Diagnosis and treatment of hip and knee osteoarthritis: a review. JAMA 325:568-578

Kelley N, Jeltema D, Duan Y, He Y (2019) The NLRP3 inflammasome: an overview of mechanisms of activation and regulation. Int J Mol Sci 20(13):3328. https://doi.org/10.3390/ijms20133328

Lisignoli G, Toneguzzi S, Piacentini A, Cristino S, Grassi F, Cavallo C, Facchini A (2006) CXCL12 (SDF-1) and CXCL13 (BCA-1) chemokines significantly induce proliferation and collagen type I expression in osteoblasts from osteoarthritis patients. J Cell Physiol 206:78-85

Liu-Bryan R (2013) Synovium and the innate inflammatory network in osteoarthritis progression. Curr Rheumatol Rep 15:323

Lu W, He Z, Shi J, Wang Z, Wu W, Liu J, Kang H, Li F, Liang S (2019) AMD3100 attenuates post-traumatic osteoarthritis by maintaining transforming growth factor-beta1-induced expression of tissue inhibitor of metalloproteinase-3 via the phosphatidylinositol 3-Kinase/Akt pathway. Front Pharmacol 10:1554

Martinon F, Tschopp J (2007) Inflammatory caspases and inflammasomes: master switches of inflammation. Cell Death Differ 14:10-22

McAllister MJ, Chemaly M, Eakin AJ, Gibson DS, McGilligan VE (2018) NLRP3 as a potentially novel biomarker for the management of osteoarthritis. Osteoarthritis Cartilage 26:612-619

Oo WM, Yu SP, Daniel MS, Hunter DJ (2018) Disease-modifying drugs in osteoarthritis: current understanding and future therapeutics. Expert Opin Emerg Drugs 23:331-347

Sandanger O, Ranheim T, Vinge LE, Bliksoen M, Alfsnes K, Finsen AV, Dahl CP, Askevold ET, Florholmen G, Christensen G, Fitzgerald KA, Lien E, Valen G, Espevik T, Aukrust P, Yndestad A (2013) The NLRP3 inflammasome is up-regulated in cardiac fibroblasts and mediates myocardial ischaemia-reperfusion injury. Cardiovasc Res 99:164-174

Scanzello CR (2017) Role of low-grade inflammation in osteoarthritis. Curr Opin Rheumatol 29:79-85
Scanzello CR, Goldring SR (2012) The role of synovitis in osteoarthritis pathogenesis. Bone 51:249-257

Sellam J, Berenbaum F (2010) The role of synovitis in pathophysiology and clinical symptoms of osteoarthritis. Nat Rev Rheumatol 6:625-635

Shen W, Chen J, Zhu T, Chen L, Zhang W, Fang Z, Heng BC, Yin Z, Chen X, Ji J, Chen W, Ouyang HW (2014) Intra-articular injection of human meniscus stem/progenitor cells promotes meniscus regeneration and ameliorates osteoarthritis through stromal cellderived factor-1/CXCR4-mediated homing. Stem Cells Transl Med 3:387-394

Shi J, Gao W, Shao F (2017) Pyroptosis: gasdermin-mediated programmed necrotic cell death. Trends Biochem Sci 42:245-254

Tschopp J, Schroder K (2010) NLRP3 inflammasome activation: the convergence of multiple signalling pathways on ROS production? Nat Rev Immunol 10:210-215

van der Kraan PM, Vitters EL, van Beuningen HM, van de Putte LB, van den Berg WB (1990) Degenerative knee joint lesions in mice after a single intra-articular collagenase injection. A new model of osteoarthritis. J Exp Pathol (Oxford) 71:19-31

Wang S, Zhou C, Zheng H, Zhang Z, Mei Y, Martin JA (2017) Chondrogenic progenitor cells promote vascular endothelial growth factor expression through stromal-derived factor-1. Osteoarthritis Cartilage 25:742-749

Wang C, Chen W, Shen J (2018) CXCR7 targeting and its major disease relevance. Front Pharmacol 9:641

Wang W, Li C, Zhang Z, Zhang Y (2019) Arsenic trioxide in synergy with vitamin $D$ rescues the defective VDR-PPAR-gamma functional module of autophagy in rheumatoid arthritis. PPAR Res 2019:6403504

Zendedel A, Johann S, Mehrabi S, Joghataei MT, Hassanzadeh G, Kipp M, Beyer C (2016) Activation and regulation of NLRP3 inflammasome by intrathecal application of SDF-1a in a spinal cord injury model. Mol Neurobiol 53:3063-3075

Zhang Y, Vasheghani F, Li YH, Blati M, Simeone K, Fahmi H, Lussier B, Roughley P, Lagares D, Pelletier JP, Martel-Pelletier J, Kapoor M (2015) Cartilage-specific deletion of mTOR upregulates autophagy and protects mice from osteoarthritis. Ann Rheum Dis 74:1432-1440

Zheng L, Zhang Z, Sheng P, Mobasheri A (2021) The role of metabolism in chondrocyte dysfunction and the progression of osteoarthritis. Ageing Res Rev 66:101249. https://doi.org/10.1016/j.arr. 2020.101249

Publisher's Note Springer Nature remains neutral with regard to jurisdictional claims in published maps and institutional affiliations. 